\title{
Spherical symmetry of generalized EYMH fields
}

\author{
H.P. Künzle and Todd A. Oliynyk ${ }^{1}$ \\ Department of Mathematical and Statistical Sciences \\ University of Alberta, Edmonton Canada T6G 2G1
}

\begin{abstract}
The possible actions of symmetry groups on generalized Higgs fields coupled to an Einstein-Yang-Mills field are studied with differential geometrical techniques involving principal and associated bundles. A classification of conjugacy classes of these actions and the form of the corresponding invariant Einstein-Yang-MillsHiggs (EYMH) fields is obtained and then applied to the case of static spherically symmetric fields over four dimensional space-time. The representations of the gauge group for which spherically symmetric Higgs fields exist are identified and the set of all field equations for the independent functions that describe these fields is analyzed and the corresponding ordinary system of differential equations is derived and shown to be consistent.
\end{abstract}

Key words: automorphisms of fiber bundles, symmetry group actions, spherical symmetry, Einstein-Yang-Mills-Higgs equations

PACS: 04.40.Nr, 11.15.Kc

\section{Introduction}

It has long been realized [1] that Yang-Mills potentials correspond to the local functions needed to describe a connection on a principal bundle $P$ over spacetime $M$ whose structure group $G_{o}$ is the physical gauge group. A local gauge transformation is then represented by a change to another local section of $P$.

Email addresses: HP.Kunzle@UAlberta.ca (H.P. Künzle), todd.oliynyk@sci.monash.edu .au (Todd A. Oliynyk ${ }^{1}$ ).

1 Present address: School of Mathematical Sciences, Monash University, VIC 3800 Australia

Preprint submitted to Elsevier Science 3 November 2018 
Similarly, while the standard Higgs field is a scalar function on space-time with values in the Lie algebra $\mathfrak{g}_{o}$ of $G_{o}$ and transforms under the adjoint transformation in $\mathfrak{g}_{o}$, it is easily generalized to have values in a vector space (or manifold) on which the gauge group acts. In fact, a generalized Higgs field is best defined as a section of a bundle $E$ associated to $P$ since that already incorporates the relation to the gauge group and the gauge changes [2-5].

Over the last one or two decades much work has been done exploring special classical solutions of Yang-Mills gauge fields in interaction with the gravitational field, the so-called Einstein-Yang-Mills (EYM) fields. This work starting with numerical regular and black hole solutions for the $S U(2)$ gauge group [6-9] and followed soon by rigorous existence proofs [10-12] has dealt mainly with the spherically symmetric static case although some very special rotationally symmetric stationary solutions have also been numerically constructed. In most papers the gauge group was chosen to be the simplest nonabelian one, namely $S U(2)$, but some numerical studies have been done for $S U(n)$ with $n \geq 3$. The inclusion of gravity unfortunately makes some of the techniques used in the Yang-Mills-Higgs theories on Minkowski space less effective like, for example, the Bogomol'nyi equations which have lead to many rigorous results for arbitrary compact gauge groups (cf. [13]. For a survey of EYM solutions see [15]1).

We have been particularly interested in studying the general geometric, analytic and algebraic problems that arise when the gauge group is an arbitrary compact semisimple Lie group and the symmetry group acts on the principal bundle by arbitrary automorphisms as long as they project onto the 'normal' action of $S O(3)$ by isometries on a static space-time manifold. In [16,17] it was shown how the the conjugacy classes of these group actions correspond to Dynkin's [18] classification of $\mathfrak{s l}_{2} \mathbb{C}$ subalgebras of semisimple Lie algebras. We then derived and analyzed to some extent the resulting system of ordinary differential equations $[17,19,20]$. It turns out that the analysis of these equations even in this static spherically symmetric case poses already many interesting problems. About the set of global solutions satisfying appropriate physical boundary conditions still very little is known for gauge groups other than $S U(2)$. The possible actions of $S U(2)$ by automorphisms on principal bundles come in two main groups which we called regular and irregular. For regular actions the static field equations allow a simple gauge choice such that with suitable boundary conditions a nonlinear and singular boundary value problem for a number of functions of the radial variable $r$ results which is numerically difficult to solve but feasible (see, for example, [21,22]). In the irregular case there appears to be no simple gauge choice to eliminate some dependent variables and the boundary value problem becomes degenerate and thus numerically quite unstable.

The purpose of this paper is two-fold. On the one hand we analyze carefully 
the possible actions of a symmetry group on both the principal and associated bundles and extend the classification of the conjugacy classes of action by automorphisms on principal bundles by Brodbeck [23] to those on associated bundles. This construction works for quite general symmetry group actions, structure groups and representations of the structure group on vector bundles subject to only very mild restrictions on the orbit structure. The main result is Theorem 1 which also allows us to make a fairly natural gauge choice in the general and in particular also the spherically symmetric case.

On the other hand we use this general result to classify the possible static spherically symmetric field equations of a general Einstein-Yang-Mills-Higgs (EYMH) system for arbitrary compact semisimple gauge groups, arbitrary symmetry group actions, and arbitrary representations defining the associated bundle whose sections are the generalized Higgs fields. This classification also applies, of course, to the Yang-Mills-Higgs theory where some early work [24] was done before the study of the EYM equations started. When generalized Higgs fields are included many results of the representation theory of compact Lie groups can be used.

We work with theories for which the Lagrange density is of the form $\mathcal{L} \sqrt{|g|} d^{4} x$ with

$$
\mathcal{L}=\varkappa R-2 \Lambda-k\left(F_{\alpha \beta}, F^{\alpha \beta}\right)-h\left(D_{\alpha} \Phi, D^{\alpha} \Phi\right)-\mathcal{W}(h(\Phi, \Phi))
$$

where $R$ is the scalar curvature of the metric $g_{\alpha \beta} d x^{\alpha} d x^{\beta}, k$ is an ad-invariant positive definite inner product on the Lie algebra $\mathfrak{g}_{o}$ of the gauge group $G_{o}$, and $h$ a (Hermitian) inner product on a (in general complex) vector space $V$, invariant under the action $\rho: G_{o} \rightarrow G L(V)$ and $\mathcal{W}$ is a scalar function of its argument serving as a potential. $D_{\alpha} \Phi$ denotes the gauge covariant derivative of the Higgs field $\Phi$ which depends on the metric, the gauge potential and $\rho$. (Also, $\varkappa=c^{4} /(8 \pi G)$ with $G$ being Newton's constant, $\Lambda$ is the cosmological constant while coupling constants for the Yang-Mills and Higgs fields can be absorbed into the definitions of the inner products $k$ and $h$.)

Part of our assumptions is that both the gauge field and the Higgs field are invariant under the appropriate actions of the symmetry group on the principal and the associated bundle, respectively. We then find that nontrivial spherically symmetric Higgs fields may not exist for certain representations $\rho$ like a 2-dimensional irreducible spinor representation, for example. This does not exclude the possibility of such a (noninvariant) Higgs field contributing to a spherically symmetric stress-energy tensor, however, and thus being compatible with a spherically symmetric space-time metric. But we are not aware of any reasonable definition of symmetry in which such Higgs fields themselves could be regarded as spherically symmetric.

The paper is organized as follows. In sections 2 and 3 we recall the definition of 
automorphisms and automorphism groups of associated bundles and establish some notation. The general classification of invariant gauge and Higgs fields under any symmetry group action is obtained in section 4 . In section 5 we specialize to the symmetry group $K=S U(2)$ and derive all field equations. We then verify that a consistent set of first and second order ordinary differential equations in the radial variable is obtained, subject to a set of constraint equations that need be satisfied only at one regular point and are then 'conserved'. This result is true whether or not the action of $K$ is regular or not, but as in the pure EYM case the gauge choice is simple only in the regular case. In the final subsection we use results from $[17,19]$ to cast the field equations into a fairly explicit form from which a numerical algorithm could be derived. We do not, in this paper, analyze what kind of boundary conditions are implied by reguarity assumptions on the solution at singular points like the center $(r=0)$ or at a black hole horizon. In the general case (arbitrary compact gauge group and arbitrary representation) this is likely to lead to a considerable number of nontrivial algebraic problems as one can guess from the experience with the EYM fields. It is, however, a necessary first step for a numerical exploration of the solution set.

\section{Associated bundles and their automorphisms}

Let $P=\left(P, \pi, M, G_{o}, R\right)$ be a principal bundle over a manifold $M$ with projection $\pi$, structure group $G_{o}$ and right action $R$ of $G_{o}$ on $P, R: P \times G_{o} \rightarrow$ $P:(p, g) \mapsto R_{g} p$.

Given another manifold $V$ and a left action $\rho: G_{o} \times V \rightarrow V$ the associated bundle $E=\left(E, P, \pi_{E}, M, G_{o}, R, \rho, V\right)$ is defined as the set of equivalence classes $[p, v]$ of elements $(p, v) \in P \times V$ with respect to the relation

$$
\left(p^{\prime}, v^{\prime}\right) \sim(p, v) \Leftrightarrow p^{\prime}=R_{g} p \text { and } v^{\prime}=\rho_{g^{-1}} v \text { for some } g \in G_{o} .
$$

We denote the fibers of $P$ and $E$ over $x \in M$ by $P_{x}=\pi^{-1}(x)$ and by $E_{x}=$ $\pi_{E}^{-1}(x)$, respectively.

We will, in general, assume that the action $\rho$ is effective, i.e. that $\rho_{g} v=$ $v \forall v \in V \Rightarrow g=e$, the identity of $G_{o}$. It then follows that $p, q \in P_{x}, \quad[p, v]=$ $[q, v] \forall v \in V \Rightarrow p=q$. Let $\hat{\pi}: P \times V \rightarrow E:(p, v) \mapsto[p, v]$ denote the canonical projection. Then $\hat{\pi}_{p}: V \rightarrow \pi_{E}^{-1}(\pi(p)): v \mapsto[p, v]$ is an isomorphism of $V$ onto $E_{\pi(p)}$ (a diffeomorphism in general, a vector space isomorphism if $V$ is a vector space). We note that $\hat{\pi}_{p}^{-1}([p, v])=v$ and also $\hat{\pi}_{R_{g} p}=\hat{\pi}_{p} \circ \rho_{g}$.

It is well known (e.g. [5]) that there is a one-to-one correspondence between the set $C \pi_{E}$ of sections $\Phi$ of $E$ and equivariant maps $\tilde{\Phi}: P \rightarrow V$ (i.e. maps 
satisfying $\left.\widetilde{\Phi} \circ R_{g}=\rho_{g^{-1}} \circ \tilde{\Phi} \forall g \in G_{o}\right)$ given by $\Phi \mapsto \widetilde{\Phi}$ with $\widetilde{\Phi}(p)=\hat{\pi}_{p}^{-1} \circ \Phi \circ \pi(p)$ and $\tilde{\Phi} \mapsto \Phi$ with $\Phi(x)=\hat{\pi}_{p} \circ \tilde{\Phi}(p)=[p, \tilde{\Phi}(p)]$ for any $p \in P_{x}$ and $x \in M$.

An automorphism of $P$ is a diffeomorphism $\psi$ of $P$ onto itself such that $\pi \circ \psi=$ $\bar{\psi} \circ \pi$ and $\psi \circ R_{g}=R_{g} \circ \psi \forall g \in G_{o}$ where $\bar{\psi}$ is an induced diffeomorphism of $M$ onto itself. An automorphism of $E$ is a bundle isomorphism $(\chi, \bar{\psi})$ of $E$, i.e. satisfying $\pi_{E} \circ \chi=\bar{\psi} \circ \pi_{E}$, and inducing an isomorphism of $E_{x}$ onto $E_{\bar{\psi}(x)}$ for any $x \in M$ that is of the form $\chi=\hat{\pi}_{q} \circ \hat{\pi}_{p}^{-1}: E_{x} \rightarrow E_{\bar{\psi}(x)}:[p, v] \mapsto[q, v]$ for a certain $p \in P_{x}:=\pi^{-1}(x)$ and $q \in P_{\bar{\psi}(x)}$ (cf. [25],p.55).

Given an automorphism $\psi$ of $P$ there is, however, a natural way to induce a related automorphism $\psi^{E}$ of an associated bundle $E$, namely by choosing $q=\psi(p)$ so that

$$
\psi^{E}=\hat{\pi}_{\psi(p)} \circ \hat{\pi}_{p}^{-1}: E_{x} \rightarrow E_{\bar{\psi}(x)}:[p, v] \mapsto[\psi(p), v] \text { for any } p \in P_{x} .
$$

In this case a section $\Phi \in C \pi_{E}$ is invariant under an automorphism $\psi_{E}$ of $E$, i.e. satisfies $\Phi \circ \bar{\psi}=\psi^{E} \circ \Phi$

iff the corresponding equivariant map $\tilde{\Phi}: P \rightarrow V$ is invariant in the sense of satisfying $\tilde{\Phi} \circ \psi=\tilde{\Phi}$.

(See [26], for example.) Every automorphism of $E$ is induced by one of $P$ in this way, provided that the action $\rho$ defining $E$ is effective.

We now describe both $\psi$ and $\psi^{E}$ with respect to a local trivialization $U \times G_{o}$ of $P$ where $U$ is an open set of $M$. Given a local section $\sigma: U \rightarrow P$ define a local trivialization of $P$ by

$$
\tau: U \times G_{o} \rightarrow \pi^{-1}(U):(x, g) \mapsto R_{g} \sigma(x) .
$$

and let

$$
\tau_{E}: U \times V \rightarrow \pi_{E}^{-1}(U):(x, v) \mapsto[\sigma(x), v]
$$

be the associated local trivialization of $E$. With respect to this trivilization a section $\Phi$ of $E$ can always be written as

$$
\Phi(x)=\tau_{E}(x, \phi(x))=[\sigma(x), \phi(x)] \quad x \in U
$$

for some map $\phi: U \rightarrow V$.

The corresponding equivariant map $\tilde{\Phi}$ then satisfies $\tilde{\Phi} \circ \tau(x, g)=\rho_{g^{-1}} \phi(x)$ and under a gauge change a Higgs field $\Phi$ transforms like $\phi(x) \mapsto \check{\phi}(x)=$

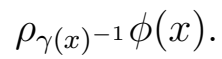

In such a local chart we can describe the automorphism $\psi$ in the form $\psi(\sigma(x))=$ $R_{\hat{\psi}(x)} \sigma(\bar{\psi}(x))$ where $\hat{\psi}: U \rightarrow G_{o}$. Then $\psi(\tau(x, g))=\tau(\bar{\psi}(x), \hat{\psi}(x) g)$. Simi- 
larly, the map $\psi^{E}([\sigma(x), v]):=\left[R_{\psi^{E}(x)} \sigma(\bar{\psi}(x)), v\right]=\left[\sigma(\bar{\psi}(x)), \rho_{\psi^{E}(x)} v\right]$ is locally of the form $\psi^{E} \circ \tau_{E}(x, v) \tau_{E}\left(\bar{\psi}(x), \rho_{\hat{\psi}(x)} v\right)$. Under a local gauge transformation $\sigma_{2}(x)=R_{\gamma(x)} \sigma_{1}(x)$ the functions $\hat{\psi}_{1}$ changes into $\hat{\psi}_{2}=\gamma\left(\bar{\psi}_{1}(x)\right)^{-1} \hat{\psi}_{1}(x) \gamma(x)$.

\section{Symmetry group acting on $P$ and $E$}

We will be interested in groups of automorphisms of $P$ and of $E$ that cover the same diffeomorphisms of $M$ and want to explore just how many independent choices can be made to describe such actions completely. For classical relativistic field theories we would expect to have an isometry group of a Lorentzian space-time manifold $M$ and, if there are gauge fields and Higgs fields present, we would expect this group to lift to act by automorphisms on the bundles $P$ and $E$ so that all physical fields are invariant under this symmetry group action. (This is to some extent even implied if Einstein's equations hold because then, if the metric is invariant, the whole stress-energy tensor will have to be invariant too which imposes strong constraints on the gauge and Higgs fields although it does not imply that they are invariant under the symmetries.)

For the remainder of this article we will assume that that the associated bundle $E=P \times_{\rho} V$ is a vector bundle. That is $V$ will be taken to be a finite dimensional vector space and $\rho: G_{o} \rightarrow \mathrm{GL}(V)$ a linear representation of $G_{o}$ on $V$. We will also assume that there is a positive definite Hermitian inner product $h: V \times V \rightarrow \mathbb{R}$ on $V$ that is invariant under the action of $G_{o}$. In other words $h\left(\rho_{g} v, \rho_{g} w\right)=h(v, w)$ for all $g \in G_{o}$ and $v, w \in V$. We note that if $G_{o}$ is compact, then there will always exist such an inner-product.

We call a principal bundle $P=P\left(M, G_{o}\right)$ on which a Lie group $K$ acts effectively on the left

$$
\psi: K \times P \longrightarrow P:(a, p) \longmapsto \psi_{a} p
$$

by principal bundle automorphisms a $K$-symmetric principal bundle. Let $\bar{\psi}$ : $K \times M \longrightarrow M:(a, x) \longmapsto \bar{\psi}_{a} x$ denote the left action of $K$ induced on $M$ via projections of $\psi_{a}$. As discussed in the previous section, the action (6) induces a natural left action of $K$ on $E$ by bundle automorphisms which is given by

$$
\psi^{E}: K \times E \longrightarrow E:(a,[p, v]) \mapsto\left[\psi_{a}(p), v\right] .
$$

and in local coordinates by

$$
\psi_{a}(x, g)=\left(\bar{\psi}_{a} x, \hat{\psi}(a, x) g\right) \quad \text { and } \quad \psi_{a}^{E}(x, v)=\left(\bar{\psi}_{a} x, \rho_{\hat{\psi}(a, x)} v\right) .
$$

The symmetry group action is therefore determined (in a given gauge) by two maps $\bar{\psi}: K \times U \rightarrow U$ and $\hat{\psi}: K \times U \rightarrow G_{o}$. The fact that $K$ acts 
on the left implies $\bar{\psi}(a b, x)=\bar{\psi}(a, \bar{\psi}(b, x)), \hat{\psi}\left(e_{K}, x\right)=e_{G_{o}}$, and $\hat{\psi}(a b, x)=$ $\hat{\psi}\left(a, \bar{\psi}_{b} x\right) \hat{\psi}(b, x)$ for all $a, b \in K$ and $x \in U$.

It is easily seen that $\psi_{E}$ induces a right action $\psi_{E}^{*}$ on the set $C \pi_{E}$ of sections of $E$ by

$$
\psi_{a}^{E *}(\Phi):=\psi_{a^{-1}}^{E} \circ \Phi \circ \bar{\psi}_{a} \quad \forall a \in K .
$$

Therefore a section $\Phi$ is called invariant under the action of $K$ if

$$
\psi_{a}^{E *}(\Phi)=\Phi \forall a \in K
$$

The invariant Hermitian inner-product $h$ can be used to induce a Hermitian inner product on the vector bundle $E$. If $\sigma: U \subset M \rightarrow P$ is a local section and $\Phi, \Psi \in C \pi_{E}$ are two sections with local representatives $\Phi^{\sigma}: U \rightarrow V$ and $\Psi^{\sigma}: U \rightarrow V$, respectively, so that

$$
\Phi(x)=\left[\sigma(x), \Phi^{\sigma}(x)\right] \quad \text { and } \quad \Psi(x)=\left[\sigma(x), \Phi^{\sigma}(x)\right] \quad \text { for all } x \in U
$$

then the Hermitian metric $h$ on $E$ is defined by the formula

$$
h(\Phi, \Phi):=h\left(\Phi^{\sigma}, \Psi^{\sigma}\right) \quad \text { for all } x \in U
$$

The $G_{o}$-invariance of $h$ guarantees that this local formula defines a global Hermitian metric.

\section{Classifying invariant Higgs fields}

As in the previous section we assume that $P=P\left(M, G_{o}\right)$ is a $K$-symmetric bundle and that $K$ acts on the vector bundle $E=P \times_{\rho} V$ according to the natural action (7). Also, for the remainder of this article we will assume that the symmetry group $K$ is compact. Once we know this, then we know that there exists an open dense subset $U \subset M$ such that $U$ is, at least locally, regularly foliated by orbits of $K$ under the action $\bar{\psi}$ on M. Fixing a point $x_{o} \in U$ and letting $K_{o}$ be the isotropy group of $x_{o}$, we then have that locally $U \approx U / K \times K / K_{o}$. This shows that we can, with a minor loss of generality, assume that $M=\widetilde{M} \times K / K_{o}$ with the $\bar{\psi}$ action given by

$$
\bar{\psi}: K \times\left(\widetilde{M} \times K / K_{o}\right) \longrightarrow \widetilde{M} \times K / K_{o}:\left(a,\left(x, k K_{o}\right)\right) \longmapsto\left(x, a k K_{o}\right)
$$

In [23] it is established that the $K$-symmetric principal bundles over base manifolds of the form $\widetilde{M} \times K / K_{o}$ can be classified by a homomorphism $\lambda: K_{o} \rightarrow G_{o}$ and a principal bundle $\widetilde{Q}$ over $\widetilde{M}$ with structure group $Z:=\operatorname{Cent}\left(\lambda\left(K_{o}\right)\right) \subset$ $G_{o}$. The classifying bundle $\widetilde{Q}$ is constructed as follows. Let $\left.P\right|_{\widetilde{M}}$ be the portion 
of $P$ over the submanifold $\widetilde{M} \cong \widetilde{M} \times\left\{e K_{o}\right\}$ (i.e. $\left.P\right|_{\widetilde{M}}:=\{p \in P: \pi(p) \in$ $\left.\left.\widetilde{M} \times e K_{o}\right\}\right)$. Then $\widetilde{M} \cong \widetilde{M} \times\left\{e K_{o}\right\}$ is a fixed point set for the action $\bar{\psi}$ and hence each fiber of $\left.P\right|_{\widetilde{M}}$ is mapped onto itself by the action of $K_{o}$ on $P$. This induces a map

$$
\mu:\left.P\right|_{\widetilde{M}} \times K_{o} \longrightarrow G_{o}:(p, h) \longmapsto \mu_{p}(h)
$$

where $\mu_{p}(h)$ is the unique element of $G_{o}$ satisfying $\psi_{h}(p)=R_{\mu_{p} h}(p)$. For each $\left.p \in P\right|_{\widetilde{M}}, \mu_{p}: K_{o} \rightarrow G_{o}$ defines a group homomorphism. Moreover, if $p,\left.q \in P\right|_{\widetilde{M}}$ are in the same fiber then the homomorphisms $\mu_{p}$ and $\mu_{q}$ belong to the same conjugacy class. Next, fix $\left.p_{o} \in P\right|_{\widetilde{M}}$ and let $\lambda:=\mu_{p_{o}}$. Then $\widetilde{Q}$ is defined by

$$
\widetilde{Q}(\widetilde{M}, Z):=\left\{\left.p \in P\right|_{\widetilde{M}}: \mu_{p}=\lambda\right\}
$$

and it can be shown that $\widetilde{Q}$ is a principal bundle over $\widetilde{M}$ with structure group $Z$. Thus each $K$-symmetric principal bundle $P$ with base manifold $M=\widetilde{M} \times$ $K / K_{o}$ determines a $Z$-bundle $\widetilde{Q}$ over $\widetilde{M}$ and a homomorphism $\lambda: K_{o} \rightarrow G_{o}$. Conversely, given $(\widetilde{Q}, \lambda)$ it is possible to construct a bundle isomorphic to $P$. We describe the construction below because it produces a principal bundle $\hat{P}$ isomorphic to $P$ on which the $K$ action is made as simple as possible. This makes it easy to identify the $K$-invariant Higgs fields.

Let $\check{P}:=\widetilde{Q} \times K$ be the product bundle with base $\widetilde{M} \times K / K_{o}$ and gauge group $\check{G}_{o}:=Z \times K_{o}$ which acts on $\check{P}$ via

$$
R_{(z, h)}(q, k)=(q, k) \cdot(z, h):=\left(R_{z}(q), k h\right) \quad \text { for all }(z, h) \in Z \times K_{o} .
$$

The projection $\pi_{\check{P}}: \check{P} \rightarrow \widetilde{M} \times K / K_{o}$ is given by $\pi_{\check{P}}(q, k)=\left(\pi_{\widetilde{Q}}(q), k K_{o}\right)$ where $\pi_{\widetilde{Q}}: \widetilde{Q} \rightarrow \widetilde{M}$ is the principal bundle projection map. Clearly,

$$
\psi^{\prime}: K \times \check{P} \rightarrow \check{P}:\left(k_{1},(q, k)\right) \mapsto\left(q, k_{1} k\right)
$$

is a left action of $K$ on $\check{P}$ by bundle automorphisms.

We let $\rho_{\lambda}$ be the homomorphism defined by $\rho_{\lambda}: \check{G}_{o} \rightarrow G_{o}:(z, h) \mapsto z \lambda(h)$, and let $\breve{G}_{o}$ act on $G_{o}$ via $\breve{G}_{o} \times G_{o} \rightarrow G_{o}:\left(g^{\prime}, g\right) \mapsto \rho_{\lambda}\left(g^{\prime}\right) g$. This allows us to define the associated bundle $\hat{P}:=\check{P} \times_{\rho_{\lambda}} G_{o}$. It can be verified that $\hat{P}$ is a principal bundle with base $\widetilde{M} \times K / K_{o}$ and structure group $G_{o}$ with the right action of $G_{o}$ given by

$$
\hat{R}_{g_{1}}([(q, k), g])=[(q, k), g] \cdot g_{1}:=\left[(q, k), g g_{1}\right] \quad g_{1} \in G_{o} .
$$

The bundle projection map $\pi_{\hat{P}}: \hat{P} \rightarrow \widetilde{M} \times K / K_{o}$ is given by $\pi_{\hat{P}}([(q, k), g]):=$ $\pi_{\check{P}}(q, k)$. As indicated above, the importance of $\hat{P}$ is that it is isomorphic to $P$ with the isomorphism defined by $\hat{P} \rightarrow P:[(q, k), g] \mapsto \psi_{k} R_{g} q$. This defines a $K$ and $G_{o}$ equivariant bundle isomorphism that induces the identity on the 
common base $\widetilde{M} \times K / K_{o}$ of the two bundles $\hat{P}$ and $P$. We also note that left action of $K$ on $\hat{P}$ is given simply by

$$
\hat{\psi}: K \times \hat{P} \longrightarrow \hat{P}:\left(k_{1},[(q, k), g]\right) \longmapsto\left[\left(q, k_{1} k\right), g\right]
$$

Now that the bundle $\hat{P}$ has been defined, we can use it to classify the invariant Higgs fields. Consider the associated vector bundle

$$
\hat{E}:=\hat{P} \times_{\rho} V
$$

with projection $\pi_{\hat{E}}: \hat{E} \rightarrow \widetilde{M} \times K / M$. The points of $\hat{E}$ are the equivalence classes $[[(q, k), g], v]$ where $[(q, k), g]$ is a point in $\hat{P}$ and $v$ is a vector in $V$. We note that the natural left $K$-action $\psi^{\hat{E}}: K \times \hat{E} \rightarrow \hat{E}$ is given by

$\psi^{\hat{E}}: K \times \hat{E} \longrightarrow \hat{E}:\left(k_{1},[[(q, k), g], v]\right) \longmapsto \psi_{k_{1}}^{\hat{E}}([[(q, k), g], v]):=\left[\left[\left(q, k_{1} k\right), g\right], v\right]$

This follows from (12) and (7). Let $\Phi: \widetilde{M} \times K / K_{o} \rightarrow \hat{E}$ be a section of $\hat{E}$ that is $K$-invariant in the sense of (9). In other words, $\Phi$ is a $K$-invariant Higgs field. From section 2, we know that $\Phi$ is equivalent to a $G_{o^{-}}$-equivariant map $\widetilde{\Phi}: \hat{P} \rightarrow V$ which satisfies $\widetilde{\Phi} \circ \hat{\psi}_{k}=\widetilde{\Phi}$ for all $k \in K$. Letting $\pi_{\check{P} \times G_{o}}$ denote the projection $\pi_{\check{P} \times G_{o}}: \check{P} \times G_{o} \rightarrow \hat{P}:(p, g) \mapsto[p, g]$, we then find that the diagram

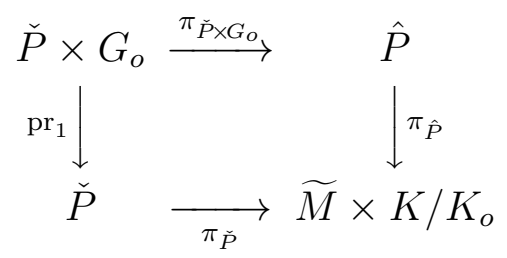

commutes. We claim that this defines a $K$-equivariant principal bundle homomorphism between $\hat{P}$ and $P$. To see this note that

$$
\pi_{\check{P} \times G_{o}}\left(k_{1} \cdot((q, k), g)\right)=\pi_{\check{P} \times G_{o}}\left(\left(\left(q, k_{1} k\right), g\right)\right)=\left[\left(q, k_{1} k\right), g\right]=k_{1} \cdot[(q, k), g]
$$

which shows that $\pi_{\check{P} \times G_{o}} \circ \psi_{k}^{\prime}=\hat{\psi}_{k} \circ \pi_{\check{P} \times G_{o}}$ for all $k \in K$, where $\psi^{\prime}:\left(k_{1},((q, k), g)\right) \mapsto$ $\left(\left(q, k_{1} k\right), g\right)$ is the natural left action of $K$ on $\check{P} \times G_{o}$. Also

$\pi_{\check{P}}\left(k_{1} \cdot(q, k)\right)=\pi_{\check{P}}\left(\left(q, k_{1} k\right)\right)=\left(\pi_{\widetilde{Q}}(q), k_{1} k K_{o}\right)=k_{1} \cdot\left(\pi_{\widetilde{Q}}(q), k K_{o}\right)=k_{1} \cdot \pi_{\check{P}}(q, k)$

which shows that $\pi_{\check{P}} \circ \psi_{k}=\bar{\psi}_{k} \circ \pi_{\check{P}}$ for all $k \in K$. This establishes the $K$ equivariance of the bundle map. We also note that since $\pi_{\check{P} \times G_{o}}\left(((q, k), g) \cdot g_{1}\right)=$ $\left[\left((q, k), g g_{1}\right]=[(q, k), g] \cdot g_{1} R\right.$, it follows that $\pi_{\check{P} \times G_{o}} \circ \check{R}_{g}=\check{R}_{g} \circ \pi_{\check{P} \times G_{o}}$ for all $g \in G_{o}$, where we are using $\check{R}_{g_{1}}((q, k), g):=\left((q, k), g g_{1}\right)$ to denote the right action of $G_{o}$ on the bundle $\check{P} \times G_{o}$. This shows that the bundle map (13) induces the identity homomorphism on $G_{o}$. 
From the commutative diagram

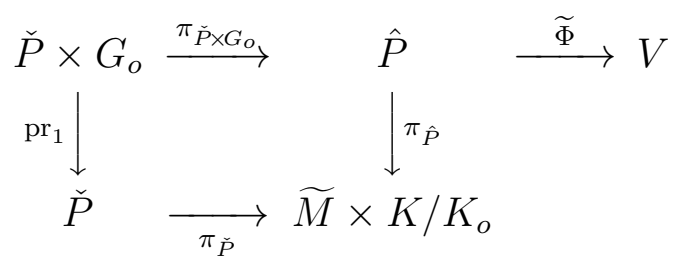

and the fact that $\pi_{\check{P} \times G_{o}}$ is surjective, it is clear that the equivariant map corresponding to the Higgs field $\hat{\Phi}$ is completely determined by the map

$$
\check{\Phi}:=\hat{\Phi} \circ \pi_{\check{P} \times G_{o}}: \check{P} \times G_{o} \longrightarrow V .
$$

Since $\pi_{\check{P} \times G_{o}}$ is both $G_{o}$ and $K$ equivariant, it follows that $\check{\Phi}$ is $K$ and $G_{o}$ equivariant. That is $\check{\Phi}$ satisfies

$$
\check{R}_{g} \circ \check{\Phi}=\rho\left(g^{-1}\right) \circ \check{\Phi} \quad \text { for all } g \in G_{o}
$$

and

$$
\check{\Phi} \circ \psi_{k}^{\prime}=\check{\Phi} \quad \text { for all } k \in K .
$$

The map $\check{\Phi}$ possesses an additional invariance coming from the construction of the bundle $\hat{P}$. Letting $\phi_{g}$ denote the action

$\phi_{g^{\prime}}: \check{P} \times G_{o} \longrightarrow \check{P} \times G_{o}:((q, k), g) \longmapsto\left(R_{g^{\prime}}((q, k)), \rho_{\lambda}\left(g^{\prime-1}\right) g\right) \quad$ for $g^{\prime} \in \check{G}_{o}$

it follows from the definition of $\hat{P}$ as the associated bundle that $\pi_{\check{P} \times G_{o}} \circ \phi_{g^{\prime}}=$ $\pi_{\check{P} \times G_{o}}$ for all $g^{\prime} \in \check{G}_{o}$. Consequently $\check{\Phi}$ satisfies

$$
\check{\Phi} \circ \phi_{g^{\prime}}=\check{\Phi} \quad \text { for all } g^{\prime} \in \check{G}_{o} \text {. }
$$

From (14) we have that $\check{\Phi}((q, k), g)=\rho_{g^{-1}} \check{\Phi}((q, k), e)$ while (15) shows that $\check{\Phi}((q, k), g)=\check{\Phi}((q, e), g)$. Combining these two results yields

$$
\check{\Phi}((q, k), g)=\rho_{g^{-1}} \check{\Phi}((q, e), e) .
$$

We also have from (16) that

$$
\check{\Phi}((q, k), g))=\rho_{g^{-1}} \check{\Phi}\left((q \cdot z, k h), z^{-1} \lambda h^{-1} g\right) .
$$

Equations (17) and (18) imply that

$$
\check{\Phi}((q \cdot z, e), e)=\rho_{z^{-1}} \rho_{\lambda\left(h^{-1}\right)} \check{\Phi}((q, e), e)
$$

for all $q \in \widetilde{Q}$ and $z \in Z, h \in K_{o}$. Defining the map

$$
\widetilde{L}: \widetilde{Q} \longrightarrow V: q \longmapsto \widetilde{L}(q):=\check{\Phi}((q, e), e),
$$


equation (17) shows that $\widetilde{L}$ and the action $\rho$ can be used to completely determine the invariant Higgs field via the relationship $\widetilde{\Phi}([(q, k), g])=\rho_{g^{-1}} \widetilde{L}(q)$ for all $q \in \widetilde{Q}, k \in K$, and $g \in G_{o}$. This can also be written as

$$
\Phi \circ \pi_{\check{P}}(q, k)=\left[[(q, k), g], \rho_{g^{-1}} \widetilde{L}(q)\right] \quad \text { for all } q \in \widetilde{Q}, k \in K \text {, and } g \in G_{o} \text {. }
$$

The map $\widetilde{L}$ is not arbitrary but is in fact a section of the associate bundle $\widetilde{Q} \times_{(\rho, Z)} V$. To see this set $h=e$ in (19) to get $\widetilde{L} \circ R_{z}=\rho_{z^{-1}} \circ \widetilde{L}$ for all $z \in Z$. This shows that $\widetilde{L}: \widetilde{Q} \rightarrow V$ is an equivariant map and hence by the discussion in section 2, uniquely determines a section of the vector bundle $\widetilde{Q} \times_{(\rho, Z)} V$. Setting $z=e$ in (19) shows that $\widetilde{L}$ must satisfy also the additional condition

$$
\rho_{\lambda(h)} \widetilde{L}(q)=\widetilde{L}(q) \quad \text { for all } q \in \widetilde{Q} \text { and all } h \in K_{o} .
$$

We summarize the above results in the following theorem:

Theorem 1 Let $P\left(M, G_{o}\right)$ be a principal bundle and suppose that

(i) $K$ is a compact Lie group that acts on $P\left(M, G_{o}\right)$ on the left by principal bundle automorphisms,

(ii) the base space $M$ is diffeomorphic to $\widetilde{M} \times K / K_{o}$ where $\widetilde{M}$ is a smooth manifold, $K_{o}$ is the isotropy subgroup of $K$ of any point $x_{o} \in M$, and the induced action of $K$ on $M$ is given by $\left(k_{1},\left(x, k K_{o}\right)\right) \rightarrow\left(x, k_{1} k K_{o}\right)$,

(iii) the $K$-symmetric bundle $P\left(M, G_{o}\right)$ is classified by a homomorphism $\lambda$ : $K_{o} \rightarrow G_{o}$ and a principal bundle $\widetilde{Q}(\widetilde{M}, Z)\left(Z:=\operatorname{Cent}\left(\lambda\left(K_{o}\right)\right) \subset G_{o}\right)$ in the sense of Brodbeck (see (11) and [23]),

(iv) $V$ is a vector space, $\rho$ is a linear representation of $G_{o}$ on $V$, and $E:=P \times_{\rho} V$ is the associated vector bundle.

Then the set of $K$-invariant sections of the vector bundle $E$ is in one-to-one correspondence with the set of maps $\widetilde{L}: \widetilde{Q} \rightarrow V$ satisfying

$$
\widetilde{L} \circ R_{z}=\rho_{z^{-1}} \circ \widetilde{L} \quad \text { and } \quad \rho_{\lambda(h)} \circ \widetilde{L}=\widetilde{L}
$$

for all $z \in Z$ and $h \in K_{o}$.

In order to derive the EYMH equations we need to have explicit local formulae for the invariant fields. This means fixing a gauge. To fix the gauge, let $\tilde{\sigma}$ : $\widetilde{U} \subset \widetilde{M} \rightarrow \widetilde{Q}$ and $\hat{\sigma}: \hat{U} \subset K / K_{o} \rightarrow K$ be two local sections. Then, if $\iota_{e}: \check{P} \rightarrow \check{P} \times G_{o}: p \mapsto(p, e)$, the two local sections $\tilde{\sigma}$ and $\hat{\sigma}$ can be used to define local section (i.e. a gauge) of $\hat{P}$ by

$$
\sigma: \widetilde{U} \times \hat{U} \subset \widetilde{M} \times K / K_{o} \rightarrow \hat{P} \quad \sigma:=\pi_{\check{P} \times G_{o}} \circ \iota_{e} \circ \tilde{\sigma} \times \hat{\sigma} .
$$

In [23] it is shown that the the set of $K$-invariant connection forms $\omega$ on $P$ is in one-to-one correspondence with the the set pairs $(\tilde{\omega}, \tilde{\Lambda})$ where $\tilde{\omega}$ is a 
connection form on $\widetilde{Q}$ and $\tilde{\Lambda}$ is a map $\tilde{\Lambda}: \widetilde{Q} \longrightarrow \mathfrak{g l}(\mathfrak{k}, \mathfrak{g})$ that satisfies

$$
\tilde{\Lambda}(q) \circ \operatorname{Ad}_{h}=\operatorname{Ad}_{\lambda(h)} \tilde{\Lambda}(q) \quad \text { and } \quad \tilde{\Lambda}(q) \cdot \xi=\lambda^{\prime}(\xi) \quad\left(\lambda^{\prime}:=T_{e} \lambda\right)
$$

for all $q \in \widetilde{Q}, h \in K_{o}$, and $\xi \in \mathfrak{k}_{o}$. Moreover, it is shown in [23] that in the gauge (23) the gauge potential $A^{\sigma}:=\sigma^{*} \omega$ can be written as $A^{\sigma}=\tilde{A}^{\sigma}+\Lambda^{\sigma} \hat{\sigma}^{*} \theta^{K}$ where $\tilde{A}^{\sigma}:=\tilde{\sigma}^{*} \tilde{\omega}, \Lambda^{\sigma}:=\tilde{\Lambda} \circ \tilde{\sigma}$, and $\theta_{h}^{K}:=T_{h} \ell_{h^{-1}}\left(\ell_{h}\right.$ being the left translation by $h$ ) is the Maurer-Cartan form on $K$. This takes care of the local formula for the gauge potential.

We now consider the Higgs field. For $\left(y, k K_{o}\right) \in \widetilde{U} \times \hat{U}$ we have $\sigma\left(y, k K_{o}\right)=$ $\left[\left(\tilde{\sigma}(y), \hat{\sigma}\left(k K_{o}\right)\right), e\right]$ and so it follows from $(21)$ that $\Phi\left(y, k K_{o}\right)=\left[\sigma\left(y, k K_{o}\right), \widetilde{L} \circ\right.$ $\tilde{\sigma}(y)]$ for all $\left(y, k K_{o}\right) \in \tilde{U} \times \hat{U}$. This shows that in the gauge (23) (see (5)) the Higgs field is given by

$$
\phi^{\sigma}\left(y, k K_{o}\right)=L^{\sigma}(y):=\widetilde{L} \circ \tilde{\sigma}(y) \quad\left(y, k K_{o}\right) \in \widetilde{U} \times \hat{U} .
$$

In view of (22), $L^{\sigma}$ must satisfy $\rho_{\lambda(h)} L^{\sigma}(y)=L^{\sigma}(y) \quad \forall h \in K_{o}, \forall y \in \widetilde{U}$. which becomes infinitesimally,

$$
\rho_{\lambda^{\prime}(\xi)} L^{\sigma}(y)=0 \quad \forall \xi \in \mathfrak{k}_{o}, \forall y \in \widetilde{U}
$$

where $\rho: \mathfrak{g}_{o} \longrightarrow \mathfrak{g l}(V)$ is the Lie algebra representation of $\mathfrak{g}_{o}$ on $V$ induced from the group representation $\rho$.

Another important fact that will be needed for the analysis of the EYMH equations is that invariant Higgs fields produce an invariant stress-energy tensor. The Higgs field contribution to the stress-energy tensor is made from the two following combinations of the Higgs field: $h(\Phi, \Phi)$ and $h\left(D_{X} \Phi, D_{Y} \Phi\right)$ where $D_{X}$ is the covariant derivative on $E$ and $h$ is the Hermitian metric on $E$ (see (10)).

Equations (8), (10), and the $G_{o}$-invariance of $h$ imply that

$$
h\left(\psi_{k}^{E} \Psi, \psi_{k}^{E} \Phi\right)=h(\Psi, \Phi)
$$

for all $(\Psi, \Phi) \in C \pi_{E} \times C \pi_{E}$ and $k \in K$. Therefore any $K$-invariant section $\Phi$ satisfies

$$
\bar{\psi}_{k}^{*}(h(\Phi, \Phi))=h(\Phi, \Phi) \quad \text { for all } k \in K .
$$

This shows that $h(\Phi, \Phi)$ defines a $K$-invariant function on $M$. 


\section{$5 \quad$ Spherically symmetric field equations}

\subsection{Field equations in general}

We assume that the Lagrange density is $\mathcal{L} \tau=\mathcal{L} \sqrt{|g|} d^{4} x$ with $\mathcal{L}$ given by (1). Since for compact Lie groups all finite-dimensional representations are equivalent to unitary ones the inner product $h$ on $V$ can be assumed to be Hermitian and positive definite. Here $\varkappa=\frac{c^{4}}{8 \pi G}$ with $G$ being Newton's gravitation constant. We may assume that any other physical coupling constants are subsumed in the choice of the inner products. (For each simple component of a semisimple gauge group $G_{o}$ and every irreducible subspace of $V$ there could be a different coupling constant.) We will also use the notation $\|X\|^{2}$ for $k(X, X)$ if $X \in \mathfrak{g}_{o}$ and for $h(X, X)$ if $X \in V$.

Variation with respect to the metric, the gauge potential components and the (real and imaginary) components of a Higgs field then yields the field equations

$$
\begin{aligned}
\varkappa\left(R_{\alpha \beta}-\frac{1}{2} R g_{\alpha \beta}\right)+\Lambda g_{\alpha \beta} & =T_{\alpha \beta} \\
k\left(A, D^{\mu} F_{\mu \alpha}\right) & =2 \operatorname{Re} h\left(\rho_{A} \Phi, D_{\alpha} \Phi\right) \quad \forall A \in \mathfrak{g}_{o} \\
D^{\mu} D_{\mu} \Phi-2 \mathcal{W}^{\prime} \Phi & =0
\end{aligned}
$$

where

$$
\begin{aligned}
T_{\alpha \beta}= & k\left(F_{\alpha \mu}, F_{\beta}{ }^{\mu}\right)-\frac{1}{4} k\left(F_{\lambda \mu}, F^{\lambda \mu}\right) g_{\alpha \beta} \\
& +h\left(D_{(\alpha} \Phi, D_{\beta)} \Phi\right)-\frac{1}{2} h\left(D_{\lambda} \Phi, D^{\lambda} \Phi\right) g_{\alpha \beta}-\frac{1}{2} \mathcal{W} g_{\alpha \beta} .
\end{aligned}
$$

Equation (29) can be written in the form

$$
D^{\mu} F_{\mu \alpha}=\tilde{\rho}\left(\Phi, D_{\alpha} \Phi\right)
$$

where $\tilde{\rho}: V \times V \rightarrow \mathfrak{g}_{o}$ is defined by

$$
\begin{aligned}
k(A, \tilde{\rho}(x, y)) & =h\left(\rho_{A} x, y\right)+h\left(y, \rho_{A} x\right) \\
& =h\left(y, \rho_{A} x\right)-h\left(x, \rho_{A} y\right) \quad \forall x, y \in V, \quad \forall A \in \mathfrak{g}_{o} .
\end{aligned}
$$

(the second formula being true because $\rho_{A}$ is an anti-Hermitian operator on $V)$. It then follows from the invariance properties of $k$ and $h$,

$$
\begin{gathered}
k([A, B], C)=k(A,[B, C]) \quad \forall A, B, C \in \mathfrak{g}_{o}, \\
h\left(\rho_{A} x, y\right)+h\left(x, \rho_{A} y\right)=0 \quad \forall A \in \mathfrak{g}_{o}, \forall x, y \in V,
\end{gathered}
$$

that the map $\tilde{\rho}$ satisfies 


$$
\begin{aligned}
\tilde{\rho}(x, y) & =-\tilde{\rho}(y, x) \\
k([A, B], \tilde{\rho}(x, y)) & =k\left(A, \tilde{\rho}\left(\rho_{B} x, y\right)\right)-k\left(B, \tilde{\rho}\left(\rho_{A} x, y\right)\right) .
\end{aligned}
$$

If $k_{\Gamma \Delta}$ and $h_{I J}$ are the components of $k$ and $h$ with respect to bases $\left\{\mathbf{e}_{\Gamma}\right\}$ of $\mathfrak{g}_{o}$ and $\left\{\mathbf{E}_{I}\right\}$ of $V$, respectively, then $\tilde{\rho}$ can be given by

$$
\tilde{\rho}_{I J}^{\Gamma}:=-2 k^{\Gamma \Sigma} \rho_{\Sigma[I}^{K} h_{J] K}
$$

where $\left(k^{\Gamma \Delta}\right)$ is the inverse matrix to $\left(k_{\Gamma \Delta}\right)$ and $\rho_{\mathbf{e}_{\Gamma}}\left(\mathbf{E}_{J}\right)=\mathbf{E}_{K} \rho_{\Gamma J}^{K}$.

In the special case where $\rho$ is the adjoint representation, the map $\tilde{\rho}$ is given by the negative of the Lie bracket, i.e. $\tilde{\rho}(A, B)=-[A, B]$.

\subsection{Spherically symmetric EYMH fields}

The spherically symmetric space-time metric can be given in a Schwarzschildlike coordinate system by

$$
g=-N S^{2} d t^{2}+N^{-1} d r^{2}+r^{2}\left(d \theta^{2}+\sin ^{2} \theta d \varphi^{2}\right) .
$$

where $N$ and $S$ are functions of $r$ and $t$, in general, and of $r$ only in the static case. The function $N$ is related to the mass function $m(r, t)$ by $N=$ $1-2 m / r-\Lambda /(3 \varkappa) r^{2}$. We assume that the space-time $M$ is diffeomorphic to $\widetilde{M} \times S^{2}$ where $\widetilde{M}$ is the 'r-t' manifold and $S^{2}$ the orbits of the symmetry group action.

The Yang-Mills potential for the gauge group $G=S U(2)$ has often been given in the so-called Witten form [27] which is, however, not easily generalized to other gauge groups. Potentials for general compact gauge groups (in the EYM case) have first been discussed by Bartnik [28] and by Brodbeck and Straumann [16]. They show that the gauge potential can be given in the form

$$
A=N S \mathcal{A} d t+\mathcal{B} d r+\boldsymbol{\Lambda}_{1} d \theta+\left(\boldsymbol{\Lambda}_{2} \sin \theta+\boldsymbol{\Lambda}_{3} \cos \theta\right) d \varphi
$$

If we choose the symmetry group to be $K=S U(2)$ whose action on spacetime has as isotropy subgroup $K_{o}=U(1)$ so that $K / K_{o} \simeq S^{2}$ then $\Lambda$ is a map from $\widetilde{M}$ into the space of linear maps from $\mathfrak{k}$ to $\mathfrak{g}_{o}$ subject to (24) which implies

$$
\left[\boldsymbol{\Lambda}_{2}, \Lambda_{3}\right]=\Lambda_{1} \&\left[\Lambda_{3}, \Lambda_{1}\right]=\Lambda_{2}
$$

where $\boldsymbol{\Lambda}_{k}=\boldsymbol{\Lambda}\left(\tau_{k}\right),\left\{\tau_{k}: k=1,2,3\right\}$ being the standard basis of $\mathfrak{s u}(2)$ with $\tau_{3}$ spanning $\mathfrak{k}_{o}$. So $\boldsymbol{\Lambda}_{3}=\lambda^{\prime}\left(\tau_{3}\right) \in \mathfrak{g}_{o}$ is a constant vector characterizing the embedding of $S U(2)$ in $G$ and thus the conjugacy class of the $S U(2)$-action on $P$. Also $\mathcal{A}$ and $\mathcal{B}$ are $\mathfrak{g}_{o}$-valued functions on $\widetilde{M}$ which, moreover, commute with $\boldsymbol{\Lambda}_{3}$. They give the "electric" part of the Yang-Mills potential. One can 
choose a temporal gauge so that $\mathcal{B}=0$, and since one is mostly interested in the noncommuting aspects of the Yang-Mills field the component $\mathcal{A}$ is often assumed to be zero, as we will also do from now on.

The static spherically symmetric field equations for the full EYMH system can now be written in a form just slightly more general than those derived in $[17,19]$. We need to observe that locally invariant Higgs fields are described by $V$-valued functions of $r$, i.e maps $r \in \tilde{U} \subset \mathbb{R} \rightarrow V$, since here $H=U(1)$, subject to the condition (25) which becomes

$$
\rho_{\boldsymbol{\Lambda}_{3}} \Phi(r)=0
$$

The Yang-Mills equations then become

$$
\begin{aligned}
r^{2} S^{-1}\left(N S \Lambda_{1}^{\prime}\right)^{\prime}-\left[\Lambda_{2}, \widehat{F}\right] & =r^{2} \tilde{\rho}\left(\Phi, \rho_{\Lambda_{1}} \Phi\right) \\
r^{2} S^{-1}\left(N S \Lambda_{2}^{\prime}\right)^{\prime}+\left[\Lambda_{1}, \widehat{F}\right] & =r^{2} \tilde{\rho}\left(\Phi, \rho_{\Lambda_{2}} \Phi\right) \\
{\left[\Lambda_{1}^{\prime}, \Lambda_{1}\right]+\left[\Lambda_{2}^{\prime}, \Lambda_{2}\right] } & =r^{2} \tilde{\rho}\left(\Phi, \Phi^{\prime}\right)
\end{aligned}
$$

where

$$
\widehat{F}:=\left[\Lambda_{1}, \Lambda_{2}\right]-\Lambda_{3}
$$

The Higgs equation takes the form

$$
S^{-1}\left(r^{2} N S \Phi^{\prime}\right)^{\prime}+\left(\rho_{\Lambda_{1}} \rho_{\Lambda_{1}}+\rho_{\Lambda_{2}} \rho_{\Lambda_{2}}\right) \Phi+\mathcal{W}^{\prime} \Phi=0 .
$$

To derive the expression for the stress-energy tensor repeated use of (41), (42), the representation property, $\rho_{[X, Y]}=\rho_{X} \rho_{Y}-\rho_{Y} \rho_{X}$ for $X, Y \in \mathfrak{g}_{o}$, as well as the assumption that $\rho_{X}$ is anti-Hermitian on $V$ must be made. We find from these relations that

$$
\begin{gathered}
{\left[\Lambda_{3}, \widehat{F}\right]=0, \quad k\left(\Lambda_{1}^{\prime}, \widehat{F}\right)=k\left(\Lambda_{2}^{\prime}, \widehat{F}\right)=0,} \\
k\left(\Lambda_{1}, \Lambda_{2}\right)=k\left(\Lambda_{1}^{\prime}, \Lambda_{2}^{\prime}\right)=k\left(\Lambda_{2}, \Lambda_{2}\right)-k\left(\Lambda_{1}, \Lambda_{1}\right)=k\left(\Lambda_{2}^{\prime}, \Lambda_{2}^{\prime}\right)-k\left(\Lambda_{1}^{\prime}, \Lambda_{1}^{\prime}\right)=0 \\
h\left(\rho_{\Lambda_{2}} \Phi, \rho_{\Lambda_{2}} \Phi\right)=h\left(\rho_{\Lambda_{1}} \Phi, \rho_{\Lambda_{1}} \Phi\right), \quad h\left(\rho_{\Lambda_{2}} \Phi, \rho_{\Lambda_{1}} \Phi\right)=-h\left(\rho_{\Lambda_{1}} \Phi, \rho_{\Lambda_{2}} \Phi\right)
\end{gathered}
$$

and then that $\left(T_{\beta}^{\alpha}\right)=\operatorname{diag}\left(-e, p_{r}, p_{\theta}, p_{\theta}\right)$ with

$$
\begin{aligned}
& e=r^{-2} N\left\|\Lambda_{1}^{\prime}\right\|^{2} \quad+\frac{1}{2} r^{-4}\|\widehat{F}\|^{2} \quad+\frac{1}{2} N\left\|\Phi^{\prime}\right\|^{2} \quad+r^{-2}\left\|\rho_{\Lambda_{1}} \Phi\right\|^{2} \quad+\frac{1}{2} \mathcal{W}, \\
& p_{r}=r^{-2} N\left\|\Lambda_{1}^{\prime}\right\|^{2} \quad-\frac{1}{2} r^{-4}\|\widehat{F}\|^{2} \quad+\frac{1}{2} N\left\|\Phi^{\prime}\right\|^{2} \quad-r^{-2}\left\|\rho_{\Lambda_{1}} \Phi\right\|^{2} \quad-\frac{1}{2} \mathcal{W}, \\
& p_{\theta}=\quad \frac{1}{2} r^{-4}\|\widehat{F}\|^{2}-\frac{1}{2} N\left\|\Phi^{\prime}\right\|^{2} \quad-\frac{1}{2} \mathcal{W} .
\end{aligned}
$$

so that the Einstein equations become

$$
\begin{aligned}
\varkappa m^{\prime} & =\frac{1}{2} r^{2} e, \\
\varkappa S^{-1} S^{\prime} & =\frac{1}{2} r N^{-1}\left(e+p_{r}\right)=r^{-1}\left\|\Lambda_{1}^{\prime}\right\|^{2}+\frac{1}{2} r\left\|\Phi^{\prime}\right\|^{2} .
\end{aligned}
$$




\subsection{Consistency of the spherically symmetric equations}

The equation (45) can be viewed as a constraint equation since the equations (43), (44), and (47) are second order differential equations which when solved will fully determine Yang-Mills potential and the Higgs field. The next proposition shows that away from the singular points where $N(r)=0, S(r)=0$, or $r=0$ the constraint equation (45) is 'conserved', i.e. automatically satisfied if it is satisfied at one point and hence it is only a constraint on the initial data for the differential equations $(43),(44),(47)$. We suspect that as in the EYM case this will still hold for solutions defined about the singular point but we have not (yet) done an analysis of the differential equation near the singular points similar to that in $[17,19]$.

Proposition 2 Suppose $\left\{N(r), S(r), \Lambda_{1}(r), \Lambda_{2}(r)\right\}$ satisfy the Yang-Mills equations (43) and (44) and the Higgs equation (47) on an interval $\left[r_{1}, r_{2}\right)\left(r_{1}>0\right)$. If neither $N(r)$ nor $S(r)$ vanish on the interval $\left[r_{1}, r_{2}\right)$ and if the constraint equation (45) holds at $r=r_{1}$ then it holds at all $r \in\left[r_{1}, r_{2}\right)$.

PROOF. Let

$$
\gamma:=\left[N S \Lambda_{1}^{\prime}, \Lambda_{1}\right]+\left[N S \Lambda_{2}^{\prime}, \Lambda_{2}\right]-\tilde{\rho}\left(\Phi, r^{2} N S \Phi^{\prime}\right)
$$

Differentiating $\gamma$ and using equations (43), (44), and (47) yields

$$
\begin{aligned}
\gamma^{\prime}=r^{-2} S( & {\left.\left[\left[\Lambda_{2}, \widehat{F}\right], \Lambda_{1}\right]-\left[\left[\Lambda_{1}, \widehat{F}\right], \Lambda_{2}\right]\right) } \\
& +S\left(\left[\tilde{\rho}\left(\Phi, \rho_{\Lambda_{1}} \Phi\right), \Lambda_{1}\right]+\left[\tilde{\rho}\left(\Phi, \rho_{\Lambda_{2}} \Phi\right), \Lambda_{2}\right]+\tilde{\rho}\left(\Phi,\left(\rho_{\Lambda_{1}}^{2}+\rho_{\Lambda_{2}}^{2}\right) \Phi\right)\right)
\end{aligned}
$$

The Jacobi identity and equation (41) imply that

$$
\left[\left[\Lambda_{2}, \widehat{F}\right], \Lambda_{1}\right]-\left[\left[\Lambda_{1}, \widehat{F}\right], \Lambda_{2}\right]=0
$$

while for any $A \in \mathfrak{g}_{o}$ and $j=1,2$,

$$
k\left(A,\left[\tilde{\rho}\left(\Phi, \rho_{\Lambda_{j}} \Phi\right), \Lambda_{j}\right]\right)=-k\left(A, \tilde{\rho}\left(\Phi, \rho_{\Lambda_{j}}^{2} \Phi\right)\right)+k\left(\Lambda_{j}, \tilde{\rho}\left(\Phi, \rho_{A} \rho_{\Lambda_{j}} \Phi\right)\right)
$$

follows from (34), (36), and (37). But

$$
\begin{aligned}
k\left(\Lambda_{j}, \tilde{\rho}\left(\Phi, \rho_{A} \rho_{\Lambda_{j}} \Phi\right)\right) & \stackrel{(33)}{=} h\left(\rho_{\Lambda_{j}} \Phi, \rho_{A} \rho_{\Lambda_{j}} \Phi\right)+h\left(\rho_{A} \rho_{\Lambda_{j}} \Phi, \rho_{\Lambda_{j}} \Phi\right) \\
& =-h\left(\rho_{A} \rho_{\Lambda_{j}} \Phi, \rho_{\Lambda_{j}} \Phi\right)+h\left(\rho_{A} \rho_{\Lambda_{j}} \Phi, \rho_{\Lambda_{j}} \Phi\right)=0
\end{aligned}
$$

since $\rho_{A}$ is anti-Hermitian. Since $A \in \mathfrak{g}_{o}$ was chosen arbitrarily, (58) then implies that

$$
\left[\tilde{\rho}\left(\Phi, \rho_{\Lambda_{1}} \Phi\right), \Lambda_{1}\right]+\left[\tilde{\rho}\left(\Phi, \rho_{\Lambda_{2}} \Phi\right), \Lambda_{2}\right]+\tilde{\rho}\left(\Phi,\left(\rho_{\Lambda_{1}}^{2}+\rho_{\Lambda_{2}}^{2}\right) \Phi\right)=0
$$


So (56), (57) and (59) imply that $\gamma^{\prime}=0$ and hence $\gamma=$ const on the interval $\left[r_{1}, r_{2}\right)$. Clearly $\gamma\left(r_{1}\right)=0$ then implies that $\gamma(r)=0$ for all $r \in\left[r_{1}, r_{2}\right)$.

It remains to investigate the consistency of the Yang-Mills equations (43) and (44) together with (41). First we have

Proposition 3 Let $\widetilde{R}_{j}:=\tilde{\rho}\left(\Phi, \rho_{\Lambda_{j}} \Phi\right)$ for $j=1,2$. Then

$$
\left[\widetilde{R}_{2}, \Lambda_{3}\right]=\widetilde{R}_{1} \quad \text { and } \quad\left[\Lambda_{3}, \widetilde{R}_{1}\right]=\widetilde{R}_{2}
$$

PROOF. Suppose $A \in \mathfrak{g}_{o}$. Then

$$
\begin{aligned}
k\left(A,\left[\Lambda_{3}, \widetilde{R}_{1}\right]\right) & =k\left(A,\left[\Lambda_{3}, \tilde{\rho}\left(\Phi, \rho_{\Lambda_{1}} \Phi\right)\right]\right) \stackrel{(34),(36)}{=}-k\left(\left[A, \Lambda_{3}\right], \tilde{\rho}\left(\rho_{\Lambda_{1}} \Phi, \Phi\right)\right) \\
& \stackrel{(37)}{=}-k\left(A, \tilde{\rho}\left(\rho_{\Lambda_{3}} \rho_{\Lambda_{1}} \Phi, \Phi\right)\right)+k\left(\Lambda_{3}, \tilde{\rho}\left(\rho_{A} \rho_{\Lambda_{1}} \Phi, \Phi\right)\right) \\
& \stackrel{(41)}{=}-k\left(A, \tilde{\rho}\left(\left(\rho_{\Lambda_{2}}+\rho_{\Lambda_{1}} \rho_{\Lambda_{3}}\right) \Phi, \Phi\right)\right)+k\left(\Lambda_{3}, \tilde{\rho}\left(\rho_{A} \rho_{\Lambda_{1}} \Phi, \Phi\right)\right) \\
& \stackrel{(42)}{=} k\left(A, \tilde{\rho}\left(\Phi, \rho_{\Lambda_{2}} \Phi\right)\right)+k\left(\Lambda_{3}, \tilde{\rho}\left(\rho_{A} \rho_{\Lambda_{1}} \Phi, \Phi\right)\right) \\
& =k\left(A, \widetilde{R}_{2}\right)+k\left(\Lambda_{3}, \tilde{\rho}\left(\rho_{A} \rho_{\Lambda_{1}} \Phi, \Phi\right)\right) .
\end{aligned}
$$

But

$$
\begin{aligned}
k\left(\Lambda_{3}, \tilde{\rho}\left(\rho_{A} \rho_{\Lambda_{1}} \Phi, \Phi\right)\right) \stackrel{(33)}{=} & h\left(\rho_{\Lambda_{3}} \rho_{A} \rho_{\Lambda_{1}} \Phi, \Phi\right)+h\left(\Phi, \rho_{\Lambda_{3}} \rho_{A} \rho_{\Lambda_{1}} \Phi\right) \\
= & -h\left(\rho_{A} \rho_{\Lambda_{1}} \Phi, \rho_{\Lambda_{3}} \Phi\right)-h\left(\rho_{\Lambda_{3}} \Phi, \rho_{A} \rho_{\Lambda_{1}} \Phi\right) \\
& \left(\text { since } \rho_{\Lambda_{3}} \text { is anti-Hermitian }\right) \\
& \stackrel{(42)}{=} 0 .
\end{aligned}
$$

Since $A \in \mathfrak{g}_{o}$ was chosen arbitrarily, the above two results imply that $\left[\Lambda_{3}, \widetilde{R}_{1}\right]=$ $\widetilde{R}_{2}$. Similar calculations show that $\left[\widetilde{R}_{2}, \Lambda_{3}\right]=\widetilde{R}_{1}$.

With (60) it follows easily that (43) and (41) together imply (44). In fact, these Yang-Mills equations are more conveniently described in complex form. Let $\mathfrak{g}=\mathfrak{g}_{o} \otimes \mathbb{C}$ be the complexification of $\mathfrak{g}_{o}$ so that $\mathfrak{g}_{o}$ is its compact real form with respect to the conjugation $c: \mathfrak{g} \rightarrow \mathfrak{g}: X+i Y \mapsto X-i Y \forall X, Y \in \mathfrak{g}_{o}$ and let

$$
\Lambda_{0}=2 i \Lambda_{3}, \quad \Lambda_{ \pm}:=\mp \boldsymbol{\Lambda}_{1}-i \boldsymbol{\Lambda}_{2}
$$

so that $\Lambda_{-}=-c\left(\Lambda_{+}\right)$and $c\left(\Lambda_{0}\right)=-\Lambda_{0}$ and, by $(41)$,

$$
\left[\Lambda_{0}, \Lambda_{ \pm}\right]= \pm 2 \Lambda_{ \pm}
$$

The Yang-Mills equations (43),(44) are then equivalent to

$$
r^{2} S^{-1}\left(N S \Lambda_{+}^{\prime}\right)^{\prime}-i\left[\widehat{F}, \Lambda_{+}\right]=-r^{2}\left(\widetilde{R}_{1}+i \widetilde{R}_{2}\right)=:-r^{2} \widetilde{R}_{+}
$$


With respect to the invariant metric $k$ the operator $\operatorname{ad}_{\Lambda_{0}}$ is Hermitian and $\mathfrak{g}$ can be decomposed into eigenspaces of $\operatorname{ad}_{\Lambda_{0}}$,

$$
\mathfrak{g}=\bigoplus \mathfrak{g}_{n}, \quad \mathfrak{g}_{n}:=\left\{X \in \mathfrak{g}:\left[\Lambda_{0}, X\right]=n X\right\}
$$

By $(62), \Lambda_{+}(r) \in \mathfrak{g}_{2} \forall r$ and therefore so are $\Lambda_{+}^{\prime}, \Lambda_{+}^{\prime \prime}$, and also, by (57), $\left[\widehat{F}, \Lambda_{+}\right]$. On the other hand, Proposition 3 implies that also the right hand side of (63) lies in $\mathfrak{g}_{2}$ for any (anti-Hermitian) representation $\rho: \mathfrak{g}_{o} \rightarrow \mathfrak{g l}(V)$ provided that the Higgs field satisfies (42). Equations (63) thus represent consistent second order differential equations for the gauge potential components, subject only to the constraints (45) being satisfied at one point.

\subsection{Explicit form of the field equations}

If we are just trying to construct a local solution of the EYMH equations in some radial interval in which none of $r, N(r)$ and $S(r)$ is zero we can choose a constant $\Lambda_{0} \in \mathfrak{g}$, subject to it being an integral lattice point within the closed fundamental Weyl chamber of some Cartan subalgebra and satisfying $c\left(\Lambda_{0}\right)=$ $-\Lambda_{0}$. (This will fix an explicit action of the symmetry group $K_{o}=S U(2)$ by automorphisms on the principal bundle [16].) Then any $r$-dependent $\Lambda_{+} \in \mathfrak{g}$ may be chosen subject to (62) and, at one point, to (45).

But the interesting and physically more relevant EYMH fields are global ones which remain regular at the center $r=0$ or at a black hole horizon where $N=0$ and which have an appropriate asymptotic behavior. It is clear from the expressions for energy density and pressures in equations (51)-(53) that $\Lambda_{1}^{\prime}, \rho_{\Lambda_{1}} \Phi$ and, in particular, $\widehat{F}$ must vanish for $r=0$. This means that also $\left[\boldsymbol{\Lambda}_{1}, \boldsymbol{\Lambda}_{2}\right]=\boldsymbol{\Lambda}_{3}$ at that point which in turn implies that the induced Lie algebra homomorphism $\lambda^{\prime}: \mathfrak{k}_{\mathfrak{o}} \rightarrow \mathfrak{g}_{o}$ defines a so-called $A_{1}$ (or defining) vector $\Lambda_{0}=$ $2 i \Lambda_{3}$ in the Cartan subalgebra of the complexified Lie algebra $\mathfrak{g}=\mathfrak{g}_{o} \otimes \mathbb{C}$ and thus a conjugacy class of $\mathfrak{s l}(2)$-subalgebras. Even when no regularity at the center is required, for example when solutions need only be found outside a black hole, natural physical fall-off conditions at infinity also imply $\widehat{F}=0$ (at least when the space-time is asymptotically flat and the magnetic charge vanishes). We will therefore from now on make the assumption that $\Lambda_{0}$ is an $A_{1}$-vector.

Up to conjugacy these $A_{1}$-vectors and their corresponding subalgebras form a finite set and, given a base $\left\{\alpha_{1}, \ldots, \alpha_{\ell}\right\}$ of the set of roots $R$ of the Lie algebra $\mathfrak{g}$, are uniquely described by the characteristic $\chi=\left(\alpha_{1}\left(\Lambda_{0}\right), \ldots, \alpha_{\ell}\left(\Lambda_{0}\right)\right)$. There is always a root base $\Delta$ such that $\alpha_{k}\left(\Lambda_{0}\right) \in\{0,1,2\} \forall k$, and all possible characteristics and thus all conjugacy classes of $\mathfrak{s l}(2))$-subalgebras of simple Lie algebras have been classified ( $[18,29])$. In view of $(62)$ the invariant connection on the principal bundle for a given conjugacy class of $K$-actions of 
automorphisms is then fully given by the (complex) functions $w_{\alpha}(r)$ such that

$$
\Lambda_{+}=\sum_{\alpha \in S_{\lambda}} w_{\alpha} \mathbf{e}_{\alpha}, \quad S_{\lambda}:=\left\{\alpha \in R: \alpha\left(\Lambda_{0}\right)=2\right\}
$$

Here we have introduced a Chevalley-Weyl basis $\left\{\mathbf{h}_{\alpha}, \mathbf{e}_{\beta}, \mathbf{e}_{-\beta}: \alpha \in \Delta, \beta \in\right.$ $\left.R^{+}\right\}$(where $R^{+}$is the set of positive roots, cf., for example, [30]) of $\mathfrak{g}$ for which we adopt the conventions and definitions 2

$$
\begin{gathered}
{\left[\mathbf{e}_{\alpha}, \mathbf{e}_{-\alpha}\right]=\mathbf{h}_{\alpha}, \quad\left[\mathbf{e}_{\alpha}, \mathbf{e}_{\beta}\right]=\nu_{\alpha, \beta} \mathbf{e}_{\alpha+\beta}, \quad \nu_{-\alpha,-\beta}=-\nu_{\alpha, \beta} \text { or } 0, \text { if } \alpha+\beta \notin R,} \\
|\alpha|^{2}:=k(\alpha, \alpha), \quad\langle\alpha, \beta\rangle:=\frac{2 k(\alpha, \beta)}{|\beta|^{2}} \quad \forall \alpha, \beta \in R, \\
k\left(\mathbf{e}_{\alpha}, \mathbf{e}_{-\alpha}\right)=-2|\alpha|^{-2} \quad \forall \alpha, \beta \in R, \quad\left(c_{i j}\right):=\left(\left\langle\alpha_{i}, \alpha_{j}\right\rangle\right) \quad \text { (Cartan matrix). }
\end{gathered}
$$

The gauge connection is thus described by as many complex functions of $r$ as there are elements in $S_{\lambda}$. In fact, by the definition of the roots, the eigenspace $\mathfrak{g}_{2}$ of $\operatorname{ad}_{\Lambda_{0}}$ is spanned by the set $\left\{\mathbf{e}_{\alpha}: \alpha \in S_{\lambda}\right\}$.

In the EYM case the field equations need to be solved for the two real functions $N$ (or $m$ ) and $S$ of $r$ and the complex functions $w_{\alpha}=\omega_{\alpha} e^{i \gamma_{\alpha}}=u_{\alpha}+i v_{\alpha}$ for $\alpha \in S_{\lambda}$. This turns out to be considerably simpler if the set $S_{\lambda}$ forms a $\Pi$ system [18], i.e. if $\alpha, \beta \in S_{\lambda}$ implies that $\alpha-\beta$ is not a root. Then $\left[\mathbf{e}_{\alpha}, \mathbf{e}_{-\beta}\right]=0$ if $\alpha$ and $\beta$ are two distinct elements of $S_{\lambda}[16,17]$. Then $S_{\lambda}$ also generates a subalgebra of $\mathfrak{g}$. In particular, $S_{\lambda}$ is a $\Pi$-system if $\Lambda_{0}$ is contained in the open Weyl chamber of the Cartan subalgebra of $\mathfrak{g}$ [16] which means, in particular, that $\alpha\left(\Lambda_{0}\right)>0 \forall \alpha \in R^{+}$. We have called this the regular case.

The simplification occurs largely because the constraint equation (45) then implies that the phase $\gamma_{\alpha}$ of $w_{\alpha}$ is constant and can be chosen zero by a gauge choice. As the following shows this may not always be the case in the presence of Higgs fields, but the equations are still much simpler.

In the following we will derive an explicit form for the Yang-Mills and the Higgs equations only since no new insight is gained by reformulating Einstein's equations.

The left hand side of equations (43)-(45) has been derived in [17] and [19].

$\overline{2}$ If the gauge group is semisimple and an invariant inner product on $\mathfrak{g}$ contains more than one 'coupling' constant this may have to be modified. 
From (65) we have

$$
\begin{aligned}
r^{2} S^{-1}\left(N S w_{\alpha}^{\prime}\right)^{\prime} & +\frac{1}{2}\left(\alpha\left(\Lambda_{0}\right) w_{\alpha}-\sum_{\beta \in S_{\lambda}}\langle\beta, \alpha\rangle\left|w_{\alpha}\right|^{2} w_{\beta}+\sum_{\beta, \gamma, \delta \in S_{\lambda}} \mu_{\alpha \delta \beta \gamma} w_{\beta} \bar{w}_{\gamma} w_{\delta}\right) \\
& =-r^{2} \widetilde{R}_{+, \alpha} \forall \alpha \in S_{\lambda}
\end{aligned}
$$

and

where

$$
\sum_{\alpha, \beta \in S_{\lambda}}\left(w_{\alpha} \bar{w}_{\beta}^{\prime}-w_{\alpha}^{\prime} \bar{w}_{\beta}\right)\left[\mathbf{e}_{\alpha}, \mathbf{e}_{-\beta}\right]=2 r^{2} \tilde{\rho}\left(\Phi, \Phi^{\prime}\right)
$$

$$
\left[\mathbf{e}_{\alpha},\left[\mathbf{e}_{\beta}, \mathbf{e}_{-\gamma}\right]\right]=: \sum_{\delta \in S_{\lambda}} \mu_{\delta \alpha \beta \gamma} \mathbf{e}_{\delta}
$$

and $\widetilde{R}_{+, \alpha}$ is the $\mathbf{e}_{\alpha}$-component of $\widetilde{R}_{+}$. Note that it follows from proposition 3 that $\widetilde{R}_{+} \in \mathfrak{g}_{2}=\operatorname{span}\left\{\mathbf{e}_{\alpha}: \alpha \in S_{\lambda}\right\}$. Moreover, $\left[\mathbf{e}_{\alpha}, \mathbf{e}_{-\beta}\right] \in \mathfrak{g}_{0}$ if $\alpha, \beta \in S_{\lambda}$ and $\tilde{\rho}\left(\Phi, \Phi^{\prime}\right) \in \mathfrak{g}_{0}$. By proposition 2 (70) needs to be solved for the $w_{\alpha}^{\prime}$ 's only for one $r$-value.

In the regular case $\mu_{\alpha \beta \gamma \delta}=0$, so (69) represents the components of an equation in the span of $\left\{\mathbf{h}_{\alpha}: \alpha \in S_{\lambda}\right\}$. Moreover, $\left[\mathbf{e}_{\alpha}, \mathbf{e}_{-\beta}\right]=\delta_{\alpha \beta} \mathbf{h}_{\alpha}$ so that (70) becomes a condition for the derivatives of the phases of the complex functions $w_{\alpha}(r)$ (which when the right hand side vanishes like in the EYM case means that the phases will be constant and the $w_{\alpha}$ s can be chosen real by fixing the gauge.)

Since in (33) the quantity $\tilde{\rho}$ is only defined for $A \in \mathfrak{g}_{o}$ in order to evaluate the right hand side of (69) and (70) we introduce (temporarily) the basis

$$
\hat{\mathbf{h}}_{j}:=-\frac{i}{2} \mathbf{h}_{j}, \hat{\mathbf{e}}_{\alpha}:=\frac{1}{2}\left(-\mathbf{e}_{\alpha}+\mathbf{e}_{-\alpha}\right), \hat{\mathbf{f}}_{\alpha}:=\frac{i}{2}\left(\mathbf{e}_{\alpha}+\mathbf{e}_{-\alpha}\right) \quad\left(j=1, \ldots \ell, \alpha \in R^{+}\right) .
$$

whose $\mathbb{R}$-linear span is the compact real form $\mathfrak{g}_{o}$ of $\mathfrak{g}$. In this basis $\left\{\mathbf{e}_{\Gamma}\right\}=$ $\left\{\mathbf{h}_{j}, \hat{\mathbf{e}}_{\alpha}, \hat{\mathbf{f}}_{\alpha}\right\}$ the invariant metric then has the form

$$
\left(\hat{k}_{\Gamma \Delta}\right)=\left(\begin{array}{ccc}
\frac{1}{2}\left|\alpha_{i}\right|^{-2} c_{i j} & 0 & 0 \\
0 & |\alpha|^{-2} \delta_{\alpha \beta} & 0 \\
0 & 0 & |\alpha|^{-2} \delta_{\alpha \beta}
\end{array}\right)
$$

We extend the anti-Hermitian representation $\rho: \mathfrak{g}_{o} \rightarrow \mathfrak{g l}(V)$ to $\mathfrak{g}$ in the obvious way, $\rho_{X+i Y}:=\rho_{X}+i \rho_{Y}$, and let

$$
\rho_{j}:=\rho_{\mathbf{h}_{j}} \forall j=1 \ldots \ell \quad \text { and } \quad \rho_{\alpha}:=\rho_{\mathbf{e}_{\alpha}} \forall \alpha \in R^{+} .
$$

It then follows for the Hermitian conjugates with respect to the inner product $h$ on $V$ that

$$
\rho_{j}^{+}=\rho_{j}, \quad \rho_{\alpha}^{+}=\rho_{-\alpha}
$$


and that $\rho_{\Lambda_{0}}$ is Hermitian and $\rho_{\Lambda_{ \pm}}^{+}=\rho_{\Lambda_{\mp}}$. Denoting the inverse of $k\left(\hat{\mathbf{h}}_{i}, \hat{\mathbf{h}}_{j}\right)$ by

$$
\hat{k}^{i j}=2 c^{i j}\left|\alpha_{j}\right|^{2}
$$

where $\left(c^{i j}\right)$ is the inverse of the Cartan matrix, we find from (33) that

$$
\begin{array}{r}
\tilde{\rho}(x, y)=-\sum_{i, j=1}^{\ell} \hat{\mathbf{h}}_{i} \hat{k}^{i j} \mathbf{I m} h\left(\rho_{j} x, y\right)-\sum_{\alpha \in R^{+}}|\alpha|^{2}\left(\boldsymbol{R e}\left[h\left(\rho_{\alpha} x, y\right)-h\left(x, \rho_{\alpha} y\right)\right] \hat{\mathbf{e}}_{\alpha}\right. \\
\left.-\operatorname{Im}\left[h\left(\rho_{\alpha} x, y\right)+h\left(x, \rho_{\alpha} y\right)\right] \hat{\mathbf{f}}_{\alpha}\right) .
\end{array}
$$

Now any (finite-dimensional) representation of $G$ is the direct sum of irreducible ones which can be obtained from irreps of $\mathfrak{g}$ and are characterized by their highest weight $\Lambda \in \mathfrak{h}^{*}$ (the dual of the Cartan subalgebra $\mathfrak{h}$ ). Any other weight $\mu$ is then given by $\mu=\Lambda-\sum_{i=1}^{\ell} q_{i} \alpha_{i}$ for certain nonnegative integers $q_{i}$. The set of eigenvalues of $\rho_{\Lambda_{0}}$ is $\mathcal{E}_{o}=\left\{\mu_{k}\left(\Lambda_{0}\right)\right\}$, where the $\mu_{k}$ are the weights of the representation. Thus spherically symmetric Higgs fields for a given $\Lambda_{0}$, i.e. choice of the action of $K$, and a given representation $\rho$ exist provided at least one of the irreducible components of $\rho$ has a weight $\mu$ with $\mu\left(\Lambda_{0}\right)=0$.

In particular, for the adjoint representation, $\rho=$ ad and $V=\mathfrak{g}_{o}$, there is always a weight 0 with multiplicity equal to the rank of $\mathfrak{g}$ and any $\Phi \in \mathfrak{h}$ is a solution of $(42)$.

Moreover, in the regular case where $\alpha\left(\Lambda_{0}\right)>0$ for all positive roots $\alpha$ every solution of (42) lies in $\mathfrak{h}$.

Next we observe that since $\rho_{\Lambda_{0}}$ is a Hermitian operator the vector space $V$ is a direct sum of mutually orthogonal eigenspaces of $\rho_{\Lambda_{0}}$,

$$
V=\bigoplus_{\sigma \in \mathcal{E}_{o}} \hat{V}_{\sigma}, \quad \hat{V}_{\sigma}:=\left\{x \in V: \rho_{\Lambda_{0}} x=\sigma x\right\}
$$

so that (42) now states that $\Phi(r) \in \hat{V}_{0} \forall r$ and thus also $\Phi^{\prime}(r) \in \hat{V}_{0}$. Moreover, it follows easily that

$$
\rho_{\alpha} V_{\sigma} \subset V_{\sigma+\alpha\left(\Lambda_{0}\right)} \quad \text { and } \quad \rho_{h} V_{\sigma} \subset V_{\sigma} \forall h \in \mathfrak{h}
$$

and therefore $\rho_{\alpha} \Phi \in \hat{V}_{2}$ when $\alpha \in S_{\lambda}$ so that, in particular,

$$
\rho_{\Lambda_{ \pm}} \Phi \in \hat{V}_{ \pm 2} \quad \text { and } \quad \rho_{j} \Phi \in \hat{V}_{0}
$$

Thus, if we replace $x$ and $y$ in (76) by $\Phi$ and $\Phi^{\prime}$, respectively, we get the expression needed on the right hand side of (70) except that the second sum 
needs only be taken over those roots $\alpha \in R^{+}$for which $\alpha\left(\Lambda_{0}\right)=0$, in view of (78), since both $\Phi$ and $\Phi^{\prime}$ lie in $\hat{V}_{0}$ and the $\hat{V}_{\sigma}$ are orthogonal for distinct $\sigma$.

In the regular case $\alpha\left(\Lambda_{0}\right)>0 \forall \alpha \in S_{\lambda}$ so that the constraint equation (70) becomes

$$
\sum_{j=1}^{\ell} \hat{k}_{i j} a_{j}\left|\alpha_{j}\right|^{2}|\alpha|^{-2} \omega_{\alpha}^{2} \gamma_{\alpha}^{\prime}=-\frac{1}{2} r^{2} \operatorname{Im} h\left(\rho_{i} \Phi, \Phi^{\prime}\right) \quad \forall i=1 \ldots \ell, \forall \alpha \in S_{\lambda}
$$

where $\alpha=\sum_{j=1}^{\ell} a_{j} \alpha_{j}$.

In the evaluation of $\widetilde{R}_{+}$one obtains expressions $h\left(\rho_{ \pm \alpha} \Phi, \rho_{ \pm \beta} \Phi\right)$ (for all choices of the signs) where $\alpha \in S_{\lambda}$ and $\beta \in R^{+}$. But if $\Phi \in \hat{V}_{0}$ then $\rho_{ \pm \alpha} \Phi \in \hat{V}_{ \pm 2}$ and $\rho_{ \pm \beta} \Phi \in \hat{V}_{ \pm \beta\left(\Lambda_{0}\right)}$. Since these eigenspaces of $\rho_{\Lambda_{0}}$ are mutually orthogonal the only inner products that are nonzero are those when $\alpha, \beta \in S_{\lambda}$ and the signs are the same. It follows that

$$
\widetilde{R}_{+}=\sum_{\alpha \in S_{\lambda}} \widetilde{R}_{+, \alpha} \mathbf{e}_{\alpha}=\sum_{\alpha \in S_{\lambda}}|\alpha|^{2} Q_{\alpha} \mathbf{e}_{\alpha}
$$

where

$$
Q_{\alpha}:=\frac{1}{2} \sum_{\beta \in S_{\lambda}}\left(R_{\alpha, \beta}+\bar{R}_{-\alpha,-\beta}\right) w_{\beta}
$$

and

$$
R_{\alpha, \beta}:=h\left(\rho_{\alpha} \Phi, \rho_{\beta} \Phi\right), \quad \alpha, \beta \in R, \Phi \in \hat{V}_{0}
$$

Again, in the regular case, or whenever we know that $\alpha, \beta \in S_{\lambda}$ implies that $\alpha-\beta$ is not a root, this simplies somewhat. For we have $\rho_{-\alpha} \rho_{\beta} \Phi=\rho_{\left[\mathbf{e}_{-\alpha}, \mathbf{e}_{\beta}\right]} \Phi+$ $\rho_{\beta} \rho_{-\alpha} \Phi=\rho_{\beta} \rho_{-\alpha} \Phi$ and therefore $R_{-\alpha,-\beta}=h\left(\rho_{-\alpha} \Phi, \rho_{-\beta} \Phi\right)=h\left(\Phi, \rho_{\alpha} \rho_{-\beta} \Phi\right)=$ $h\left(\Phi, \rho_{-\beta} \rho_{\alpha} \Phi\right)=h\left(\rho_{\beta} \Phi, \rho_{\alpha} \Phi\right)=\overline{h\left(\rho_{\alpha} \Phi, \rho_{\beta} \Phi\right)}=\bar{R}_{\alpha, \beta}$ so that

$$
Q_{\alpha}:=\sum_{\beta \in S_{\lambda}} R_{\alpha, \beta} w_{\beta}
$$

\section{Conclusions}

We have shown how to, in principle, construct Einstein-Yang-Mills-Higgs systems that are invariant under an arbitrary action of a space-time symmetry group that acts by principle bundle automorphisms which leave the gauge connection invariant as well as Higgs fields defined via any unitary representation of the (compact) gauge group. The classification of the possible actions by automorphisms is known for the symmetry group $S U(2)$, but may be more difficult to find for larger groups. One would need to first find all conjugacy classes of a certain type of Lie subalgebras of the gauge Lie algebra. 
We have obtained an explicit form of the full field equations in the static spherically symmetric case and shown that they form a consistent system of ordinary differential equations. Before global solutions can be found numerically it would be necessary to investigate in some detail the boundary conditions that regularity conditions at a center, horizon or in an asymptotic region will imply.

It must be pointed out that not all cases of physical interest even for the static spherically symmetric case are covered by this approach. For example, the doublet Higgs field coupled to an $S U(2)$-gauge and gravitational field in [31] cannot be described in our formalism because the Higgs field is not spherically symmetric. In fact, the representation of $\mathfrak{s u}(2)$ in this case is the direct sum of two irreducible two-dimensional ones for which there is no weight $\mu$ with $\mu\left(\Lambda_{0}\right)=0$. These authors make a simple ansatz for the Higgs field using the gauge choice for the potential often attributed to Witten [27]. They then find that the stress-energy tensor is spherically symmetric and thus compatible with a spherically symmetric ansatz for the space-time metric.

One might ask whether with our gauge choice one can assume that only the quantity $h\left(D_{(\alpha} \Phi, D_{\beta} \Phi\right)$ is spherically symmetric rather than $\Phi$ itself. Unfortunately this is not possible since then $h\left(\rho_{\Lambda_{3}} \Phi, \rho_{\Lambda_{3}} \Phi\right)$ would have to vanish which implies $\rho_{\Lambda_{3}} \Phi=0$, i.e. an invariant Higgs field. On the other hand we do not know whether the Witten ansatz for spherically symmetric gauge fields can be generalized to gauge groups other than $S U(2)$ or whether perhaps another equally convenient gauge exists.

\section{References}

[1] C. N. Yang, T. T. Wu, Concept of nonintegrable phase factors and global formulation of gauge fields, Phys. Rev. D (3) 12 (1975) 3845-3857.

[2] A. Trautman, Fiber bundles, gauge fields, and gravitation, Plenum Press New York, 1980, pp. 287-308.

[3] P. Forgács, N. Manton, Space-time symmetries in gauge theories, Comm. Math. Phys. 72 (1980) 15-35.

[4] J. Harnad, S. Shnider, L. Vinet, Group actions on principal bundles and invariance conditions for gauge fields, J. Math. Phys. 21 (1980) 2719-2724.

[5] K. Marathe, G. Martucci, Mathematical foundations of gauge theories, Elsevier, Amsterdam, 1992.

[6] R. Bartnik, J. Mckinnon, Particlelike solutions of the Einstein-Yang-Mills equations, Phys. Rev. Lett. 61 (1988) 141-144. 
[7] H. Künzle, A. Masood-ul-Alam, Spherically symmetric static $S U(2)$ EinsteinYang-Mills fields, J. Math. Phys. 21 (1990) 928-935.

[8] M. Volkov, D. Gal'tsov, Black holes in Einstein-Yang-Mills theory, Soviet J. Nuclear Phys. 51 (1990) 747-753.

[9] P. Bizoń, Colored black holes, Phys. Rev. Lett. 64 (1990) 2844-2847.

[10] J. Smoller, A. Wasserman, S.-T. Yau, J. McLeod, Smooth static solutions of the Einstein/Yang-Mills equations, Comm. Math. Phys. 143 (1991) 115-147.

[11] J. Smoller, A. Wasserman, S.-T. Yau, Existence of black hole solutions for the Einstein-Yang/Mills equations, Comm. Math. Phys. 154 (1993) 377-401.

[12] P. Breitenlohner, P. Forgács, D. Maison, Static spherically symmetric solutions of the Einstein-Yang-Mills equations, Comm. Math. Phys. 163 (1994) 141-172.

[13] A. Jaffe, C. Taubes, Vortices and monopoles (Structure of static gauge theories), Birkhäuser, Boston, 1980.

[14] A. Marini, L. Sadun, Spherically symmetric solutions of a boundary value problem for monopoles, J. Math. Phys. 44 (2003) 1071-1083.

[15] M. Volkov, D. Gal'tsov, Gravitating non-Abelian solitons and black holes with Yang-Mills fields, Phys. Rep. 319 (1999) 1-83.

[16] O. Brodbeck, N. Straumann, A generalized Birkhoff theorem for the EinsteinYang-Mills system, J. Math. Phys. 34 (1993) 2412-2423.

[17] T. Oliynyk, H. Künzle, Local existence proofs for the boundary value problem for static spherically symmetric Einstein-Yang-Mills fields with compact gauge groups, J. Math. Phys. 43 (2002) 2363-2393.

[18] E. Dynkin, Semisimple subalgebras of semisimple Lie algebras, Amer. Math. Soc. Transl. (2)6 (1957) 111-244.

[19] T. Oliynyk, H. Künzle, On all possible static spherically symmetric EYM solitons and black holes, Classical Quantum Gravity 19 (2002) 457-482.

[20] T. Oliynyk, H. Künzle, On global properties of static spherically symmetric EYM fields with compact gauge groups.

[21] B. Kleihaus, J. Kunz, A. Sood, SU(3) Einstein-Yang-Mills sphalerons and black holes, Phys. Lett. B 354 (1995) 240-246.

[22] B. Kleihaus, J. Kunz, A. Sood, Charged $S U(N)$ Einstein-Yang-Mills black holes, Phys. Lett. B 418 (1998) 284-293.

[23] O. Brodbeck, On symmetric gauge fields for arbitrary gauge and symmetry groups, Helv. Phys. Acta (1996) 321-324.

[24] C. Gu, H. Hu, On the spherically symmetric gauge fields, Comm. Math. Phys. 79 (1981) 75-90. 
[25] S. Kobayashi, K. Nomizu, Foundations of differential geometry I, Interscience, Wiley, New York, 1963.

[26] A. Trautman, Geometrical aspects of gauge configurations, Acta Phys. Austriaca Suppl. 23 (1981) 401-432.

[27] E. Witten, Some exact multipseudoparticle solutions in classical Yang-Mills theory, Phys. Rev. Lett. 38 (1977) 121-124.

[28] R. Bartnik, The spherically symmetric Einstein Yang-Mills equations, in: Z. Perjés (Ed.), Relativity Today, 1989, Tihany, Nova Science Pub., Commack NY, 1992, pp. 221-240, proceedings of the third Hungarian relativity workshop, Tihany 1989.

[29] D. Collingwood, W. McGovern, Nilpotent orbits in semisimple Lie algebras, Van Nostrand Reinhold, New York, 1993.

[30] J. Humphreys, Introduction to Lie algebras and representation theory, Springer New York, 1972.

[31] B. Greene, S. Mathur, C. O'Neill, Eluding the no-hair conjecture: Black holes in spontaneously broken gauge theories, Phys. Rev. D (3) 47 (1993) 2242-2259. 